\title{
Vibration Characteristics of Axially Moving Titanium- Polymer Nanocomposite Faced Sandwich Plate Under Initial Tension
}

\author{
Ali Ghorbanpour Arani ${ }^{a^{*}}$, Elham Haghparast ${ }^{a}$ and Hassan BabaAkbar Zarei ${ }^{a}$ \\ ${ }^{a}$ Faculty of Mechanical Engineering, Institute of Nanoscience\& Nanotechnology, University of Kashan, Kashan, \\ Iran \\ ${ }^{*}$ E-mail address: aghorban@kashanu.ac.ir
}

Received date: March 2017

\begin{abstract}
In the present research, vibration and instability of axially moving sandwich plate made of soft core and composite face sheets under initial tension is investigated. Single-walled carbon nano-tubes (SWCNTs) are selected as a reinforcement of composite face sheets inside Poly methyl methacrylate (PMMA) matrix. Higher order shear deformation theory (HSDT) is utilized due to its accuracy of polynomial functions than other plate theories. Based on extended rule of mixture, the structural properties of composite face sheets are taken into consideration. Motion equations are obtained by means of Hamilton's principle and solved analytically. Influences of various parameters such as axially moving speed, volume fraction of CNTs, pre-tension, thickness and aspect ratio of sandwich plate on the vibration characteristics of moving system are discussed in details. The results indicated that the critical speed of moving sandwich plate is strongly dependent on the volume fraction of CNTs. Therefore, the critical speed of moving sandwich plate can be improved by adding appropriate values of CNTs. The results of this investigation can be used in design and manufacturing of marine vessels and aircrafts.
\end{abstract}

Keywords: Vibration analysis; Axially moving; sandwich plate; Nanocomposite face sheets, Initial tension.

\section{Introduction}

The use of sandwich structures in the world is increasingly growing. In today's modern engineering, sandwich structures are being used successfully for a variety of applications such as aircraft, wind turbine blades, spacecraft, train and car structures, boat/ship hulls boat/ship superstructures and many others. This is due to the excellent mechanical properties of these structures (High strength to weight ratio, high resistance to impact, flexibility and etc.). Most of sandwich structures are composed of three layers: the top layer, middle layer that is called the core and the bottom layer. The core is less stiff compared to other two-layer. Hence, selecting the appropriate material for the core and the other layer is a significant for optimum design of sandwich structures. Carbon nanotube-reinforced composite can be an excellent option for the top and bottom layers due to the high stiffness and the other supreme properties. In this regard, study on vibration and instability of sandwich structures which are reinforced by carbon fibers have been conducted by many researchers that some of them are presented below.

Thostenson and Chou [1] have modelled the elastic properties of carbon nanotube-reinforced composite. Investigation of the structure/size influence of carbon nanotubes on the elastic properties 
of nanotube-based composites is the main objective of their research. Zhou et al. [2] analyzed the static and free vibration of carbon nanotube-reinforced composite plates using finite element method with first order shear deformation plate theory (FSDT). They have studied on the influences of the volume fractions of carbon nanotubes and the edge-to-thickness ratios on the bending responses, natural frequencies and mode shapes of the plates. Also, Lei et al. [3] have done similar work before, but they used the element free kP-Ritz method in thermal environment. Bending behavior of functionally graded carbon nanotube reinforced composite (FG-CNTRC) plate embedded in thin piezoelectric layers subjected to mechanical uniform load is investigated by Alibeigloo [4]. He applied simply supported boundary conditions on plate and used three-dimensional elasticity theory to analyze bending behavior of composite plate.

In recent years, with the advance of industry, there was a need for structures with multiple capabilities simultaneously. One of the requirements was answered by the discovery of sandwich structures. Thus, researchers have been working in this field. Nayak et al. [5] investigated free vibration analysis of composite sandwich plates based on Reddy's higher-order theory. Using this theory that they have provided, it can be calculated the natural frequencies of isotropic, orthotropic, and layered anisotropic composite and sandwich plates. Utilizing radial basis collocation function, Ferreira et al. [6] analyzed the static, buckling and vibration responses of the plate. Khalili and Mohammadi [7] used improved high-order sandwich plate to analyze the free vibration of sandwich plates with FG face sheets. The material properties of FG face sheets and core are considered to be temperature-dependent by a third-order function of temperature. Recently, Sahoo and Singh [8] proposed a new trigonometric zigzag theory to analyze the static analysis of laminated composite and sandwich plates. They assumed shear strain shape function for non-linear distribution of in-plane displacement across the thickness. Thai et al. [9] presented a new first-order shear deformation theory for functionally graded sandwich plates composed of isotropic core and functionally graded face sheets. They approved that the presented theory is accurate in predicting the bending, buckling and free vibration responses of FG sandwich plates. In another work, Plagianakos and Papadopoulos [10] presented coupled higher-order layerwise piezoelectric laminate mechanics. Their developed model was applicable to predict the static electromechanical response of composite and sandwich composite plates subjected to static mechanical loads and/or electric voltages. Natarajan et al. [11] have attempted to achieve an efficient solution for the bending and free vibration analysis of sandwich plates with CNT reinforced composite face sheets. For this purpose, they have used QUAD-8 shear flexible element developed based on higher-order structural theory. This theory considered the possible discontinuity in slope at the interfaces layers, the realistic variation of the displacements through the thickness, and the thickness stretch effects on the transverse deflection. Kheirikhah et al. [12] carried out biaxial buckling analysis of soft-core composite sandwich plates. In this way, they employed third-order plate theory for face sheets and quadratic and cubic functions for transverse and in-plane displacements of the core, respectively. Moreover, analytical solution has been presented for sandwich plates with simply supported boundary conditions under biaxial inplane compressive loads using Navier's solution.

Axially moving beams and plates have attracted many authors. The geometrically nonlinear dynamics and stability of an axially moving plate is presented by Ghayesh et al. [13]. In their study, plate is placed under an out-of-plane incitement load and the frequency-response curves of the system are plotted. Also, Dong Yang et al. [14] have been working on the previous thread. To solve the differential equations governing the problem, they have used both the Galerkin method and differential quadrature method. In the case of free vibration analysis of axially moving viscoelastic plates, Hatami et al. [15] and Marynowski [16] have studied. However, each of them has used different models for their work. Marynowski and Grabski [17] have investigated dynamic analysis of an axially moving plate subjected to thermal loading using the extended Galerkin method the. In 
addition, they have been examined the effects of transport speed, the thermal critical loading and axial tension on dynamic behavior of axially moving aluminum plate.

Despite mentioned researches, vibration and instability analysis of axially moving sandwich plate under initial tension using HSDT is a novel topic that cannot be found in literature. To the best of authors' knowledge, for the first time, analysis of axially moving sandwich plate with CNT face sheets is developed in this paper. Material properties of composite plate are obtained based on extended rule of mixture. Motions equations are obtained based on energy method and solved by means of analytical approach. Influences of various parameters such as moving speed, volume fraction of CNTs, pre-tension load, thickness and aspect ratio on instability and critical speed of moving composite sandwich plate are discussed in details. To verify the presented method, the natural frequencies for stationary sandwich plate have been compared with previous researches. The result of this work can be useful to control and improve the performance of axially moving devices which are employed in military equipment.

\section{Potential energies of axially moving sandwich plate}

Consider a rectangular sandwich plate with length $(a)$, width $(b)$ and thickness $\left(h=h^{t}+h^{c}+h^{b}\right)$ which is shown in Fig.1. The top and bottom layers are made of carbon nanotube-reinforced composite plate. The carbon nanotube is distributed uniformly in the $\mathrm{x}$ direction. The Cartesian coordinate system is selected for this problem. $x$ and $y$ axes are located in the mid-plane and $z$ axis located along the thickness direction. Sandwich plate is moving along the $x$ direction with the constant velocity $V$.

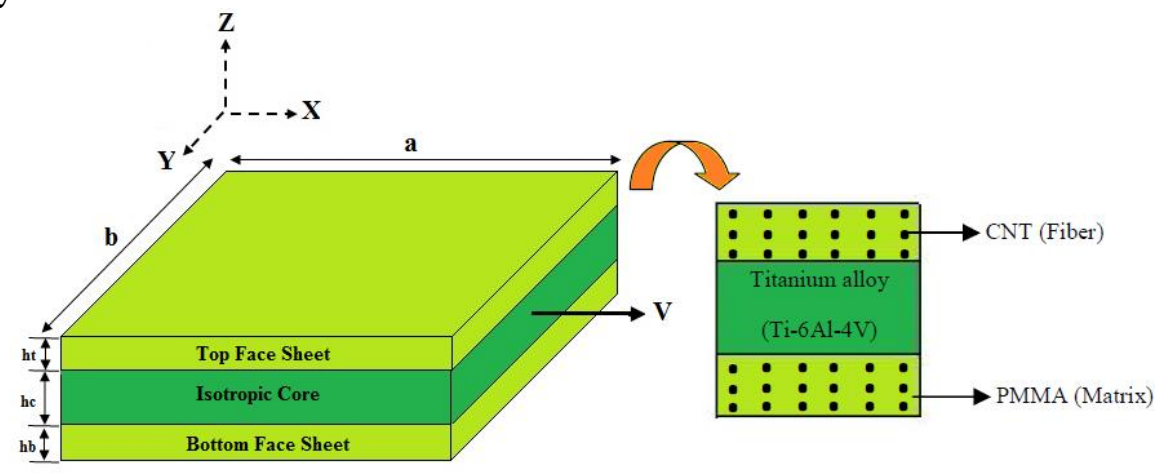

Fig. 1. Schematic figure of axially moving sandwich plate with CNT reinforced face sheets.

The following assumptions have been used to derive motion equations [18 and 19]:

- The core thickness is larger and softer than the top and bottom layer.

- The core is fully bonded with the top and bottom layers. Thus, core and the top layer have the same displacement in $\left(z=+h^{c} / 2\right)$ as well as the core and the bottom layer in $\left(z=-h^{c} / 2\right)$,

- No slipping happens at the interfaces between the three layers of the sandwich plate.

Because the core is made of a soft material, to increase the accuracy of results a higher-order theory will be used. According to this theory, the displacement field of the sandwich plate can be expressed as [20]: 


$$
\begin{aligned}
& \mathrm{u}(\mathrm{x}, \mathrm{y}, \mathrm{z}, \mathrm{t})=\mathrm{u}_{0}(\mathrm{x}, \mathrm{y}, \mathrm{t})+\mathrm{zu}_{1}(\mathrm{x}, \mathrm{y}, \mathrm{t})+\mathrm{z}^{2} \mathrm{u}_{2}(\mathrm{x}, \mathrm{y}, \mathrm{t})+\mathrm{z}^{3} \mathrm{u}_{3}(\mathrm{x}, \mathrm{y}, \mathrm{t}), \\
& \mathrm{v}(\mathrm{x}, \mathrm{y}, \mathrm{z}, \mathrm{t})=\mathrm{v}_{0}(\mathrm{x}, \mathrm{y}, \mathrm{t})+\mathrm{zv}_{1}(\mathrm{x}, \mathrm{y}, \mathrm{t})+\mathrm{z}^{2} \mathrm{v}_{2}(\mathrm{x}, \mathrm{y}, \mathrm{t})+\mathrm{z}^{3} \mathrm{v}_{3}(\mathrm{x}, \mathrm{y}, \mathrm{t}), \\
& \mathrm{w}(\mathrm{x}, \mathrm{y}, \mathrm{z}, \mathrm{t})=\mathrm{w}_{0}(\mathrm{x}, \mathrm{y}, \mathrm{t})+\mathrm{zw}_{1}(\mathrm{x}, \mathrm{y}, \mathrm{t})+\mathrm{z}^{2} \mathrm{w}_{2}(\mathrm{x}, \mathrm{y}, \mathrm{t}),
\end{aligned}
$$

in which, $u_{j}, v_{j}$ and $w_{k}(j=0,1,2,3$ and $k=0,1,2)$ are the unknowns of the displacement components of the sandwich plate. In this manner, eleven displacements are unknowns.

The linear von-Karman strain-displacement relations can be defined as:

$$
\begin{gathered}
\varepsilon_{\mathrm{xx}}^{\mathrm{i}}=\mathrm{u}_{, \mathrm{x}}=\mathrm{u}_{0, \mathrm{x}}+\mathrm{zu}_{1, \mathrm{x}}+\mathrm{z}^{2} \mathrm{u}_{2, \mathrm{x}}+\mathrm{z}^{3} \mathrm{u}_{3, \mathrm{x}}, \\
\varepsilon_{\mathrm{yy}}^{\mathrm{i}}=\mathrm{v}_{, \mathrm{y}}=\mathrm{v}_{0, \mathrm{y}}+\mathrm{zv}_{1, \mathrm{y}}+\mathrm{z}^{2} \mathrm{v}_{2, \mathrm{y}}+\mathrm{z}^{3} \mathrm{v}_{3, \mathrm{y}}, \\
\varepsilon_{\mathrm{zz}}^{\mathrm{i}}=\mathrm{w}_{, \mathrm{z}}=\mathrm{w}_{1}+2 \mathrm{zw}_{2}, \\
\varepsilon_{x y}^{i}=\frac{1}{2}\left(\mathrm{v}_{, \mathrm{x}}+\mathrm{u}_{, \mathrm{y}}\right)=\frac{1}{2} \mathrm{v}_{0, \mathrm{x}}+\frac{1}{2} z \mathrm{v}_{1, \mathrm{x}}+\frac{1}{2} z^{2} \mathrm{v}_{2, \mathrm{x}}+\frac{1}{2} z^{3} \mathrm{v}_{3, \mathrm{x}}+\frac{1}{2} \mathrm{u}_{0, \mathrm{y}}+\frac{1}{2} z \mathrm{u}_{1, \mathrm{y}}+\frac{1}{2} z^{2} \mathrm{u}_{2, \mathrm{y}}+\frac{1}{2} z^{3} \mathrm{u}_{3, \mathrm{y}}, \\
\varepsilon_{x z}^{i}=\frac{1}{2}\left(\mathrm{w}_{, \mathrm{x}}+\mathrm{u}_{, \mathrm{z}}\right)=\frac{1}{2} \mathrm{w}_{0, \mathrm{x}}+\frac{1}{2} z \mathrm{w}_{1, \mathrm{x}}+\frac{1}{2} z^{2} \mathrm{w}_{2, \mathrm{x}}+\frac{1}{2} \mathrm{u}_{1}+\mathrm{zu}_{2}+\frac{3}{2} \mathrm{z}^{2} \mathrm{u}_{3}, \\
\varepsilon_{y z}^{i}=\frac{1}{2}\left(\mathrm{w}_{, \mathrm{y}}+\frac{\partial}{\partial \mathrm{z}} \mathrm{v}_{, \mathrm{z}}\right)=\frac{1}{2} \mathrm{w}_{0, \mathrm{y}}+\frac{1}{2} z \mathrm{w}_{1, \mathrm{y}}+\frac{1}{2} z^{2} \mathrm{w}_{2, \mathrm{y}}+\frac{1}{2} \mathrm{v}_{1}+\mathrm{zv}_{2}+\frac{3}{2} \mathrm{z}^{2} \mathrm{v}_{3},
\end{gathered}
$$

where $\varepsilon_{p q}^{i}(p, q=x, y, z$ and $i=t, c, b)$ is strain of $i$ th layers. It is obvious that all layers have the same strain due to considering similar displacement field for all of them. The constitutive equations for sandwich plate can be obtained as [12]:

$$
\left\{\begin{array}{c}
\sigma_{x x}^{i} \\
\sigma_{y y}^{i} \\
\sigma_{z z}^{i} \\
\sigma_{y z}^{i} \\
\sigma_{x z}^{i} \\
\sigma_{x y}^{i}
\end{array}\right\}=\left[\begin{array}{cccccc}
Q_{11}^{i} & Q_{12}^{i} & Q_{13}^{i} & 0 & 0 & 0 \\
Q_{12}^{i} & Q_{22}^{i} & Q_{23}^{i} & 0 & 0 & 0 \\
Q_{13}^{i} & Q_{23}^{i} & Q_{33}^{i} & 0 & 0 & 0 \\
0 & 0 & 0 & Q_{44}^{i} & 0 & 0 \\
0 & 0 & 0 & 0 & Q_{55}^{i} & 0 \\
0 & 0 & 0 & 0 & 0 & Q_{66}^{i}
\end{array}\right]\left\{\begin{array}{c}
\varepsilon_{x x}^{i} \\
\varepsilon_{y y}^{i} \\
\varepsilon_{z z}^{i} \\
\varepsilon_{y z}^{i} \\
\varepsilon_{x z}^{i} \\
\varepsilon_{x y}^{i}
\end{array}\right\},
$$

where $\sigma_{p q}^{i}$ and $Q_{r s}^{i}(r, s=1,2,3$ and 44,55,66) are stress and the stiffness coefficient matrix of $i$ th layers, respectively. In this paper, the stiffness coefficients is defined for plain strain problems with isotropic core $\left(Q_{r s}^{c}\right)$, orthotropic top and bottom layers $\left(Q_{r s}^{t, b}\right)$. Also, the extended rule of mixture is used to calculate mechanical properties of CNTRC face sheets [12]:

$$
\begin{gathered}
Q_{11}^{i}=\frac{E_{11}^{i}}{1-v_{12}^{i} v_{21}^{i}}, \quad Q_{12}^{i}=\frac{v_{12}^{i} E_{11}^{i}}{1-v_{12}^{i} v_{21}^{i}}, \quad Q_{21}^{i}=\frac{v_{21}^{i} E_{11}^{i}}{1-v_{12}^{i} v_{21}^{i}}, \quad Q_{22}^{i}=\frac{E_{22}^{i}}{1-v_{12}^{i} v_{21}^{i}}, \\
Q_{44}^{i}=G_{23}^{i}, \quad Q_{55}^{i}=G_{13}^{i}, \quad Q_{66}^{i}=G_{12}^{i},
\end{gathered}
$$

where:

$$
E_{11}^{i}=\eta_{1} V_{f}^{i} E_{11 f}^{i}+V_{m}^{i} E_{m}^{i}
$$




$$
\begin{aligned}
& \frac{\eta_{2}}{E_{22}^{i}}=\frac{V_{c n t}^{*}}{E_{22 f}^{i}}+\frac{V_{m}^{i}}{E_{m}^{i}}, \\
& \frac{\eta_{3}}{G_{12}^{i}}=\frac{V_{c n t}^{*}}{G_{12 f}^{i}}+\frac{V_{m}^{i}}{G_{m}^{i}} .
\end{aligned}
$$

The total potential energy consists of two factors, bending and elongation. Thus, it can be written as:

$$
U^{i}=U_{b}^{i}+U_{e}^{i}
$$

where $U_{b}^{i}$ and $U_{e}^{i}$ represent potential energy due to bending and elongation, respectively, and defined as [21]:

$$
\begin{gathered}
U_{b}^{i}=\int_{V^{i}}\left[\frac{1}{2}\left(\sigma_{x x}^{i} \varepsilon_{x x}^{i}+\sigma_{y y}^{i} \varepsilon_{y y}^{i}+\sigma_{z z}^{i} \varepsilon_{z z}^{i}\right)+\sigma_{x y}^{i} \varepsilon_{x y}^{i}+\sigma_{x z}^{i} \varepsilon_{x z}^{i}+\sigma_{y z}^{i} \varepsilon_{y z}^{i}\right] d V, \\
U_{e}^{i}=\int_{V} \frac{1}{2} \sigma_{x x}^{0}\left(w_{, x}\right)^{2} d V,
\end{gathered}
$$

in which, $\sigma_{x x}^{0}$ represent the uniform initial stress along the x direction. Hence, it is neglected the shear stress and the normal stress of the uniform initial stress in the y direction.

\section{Kinetic energy}

The velocity vector $(\vec{V})$ for axially moving sandwich plate with constant velocity $C$ can be expressed as follows [13]:

$$
\vec{V}=\left(C+u_{, t}+C u_{, x}\right) \vec{i}+\left(v_{, t}+C v_{, x}\right) \vec{j}+\left(w_{, t}+C w_{, x}\right) \vec{k}
$$

Thus, the kinetic energy of the sandwich plates is given by:

$$
K^{i}=\frac{1}{2} \rho^{i} \int_{V^{i}}\left[\left(\mathrm{C}+\mathrm{u}_{, \mathrm{t}}+\mathrm{Cu}_{, \mathrm{x}}\right)^{2}+\left(\mathrm{v}_{, \mathrm{t}}+\mathrm{Cv}_{, \mathrm{x}}\right)^{2}+\left(\mathrm{w}_{, \mathrm{t}}+\mathrm{Cw}, \mathrm{x}\right)^{2}\right] d V
$$

where $K^{i}$ and $\rho^{i}$ represent kinetic energy and density of $i$ th layers, respectively.

\section{Motion equations based on Hamilton's principle}

Based on Hamilton's principle, equations of motion for axially moving sandwich plate are derived as [21]:

$$
\delta \Pi=\delta \int_{t_{1}}^{t_{2}}\left(U^{i}-K^{i}\right) d t=0
$$


A. Ghorbanpour Arani, E. Haghparast, H. BabaAkbar Zarei

Substituting Eqs. (7a), (7b) and (9) into Eq. (10), the coefficients of $\delta u_{0}, \delta u_{1}, \delta u_{2}, \delta u_{3}, \delta v_{0}, \delta v_{1}, \delta v_{2}, \delta v_{3}, \delta w_{0}, \delta w_{1}$ and $\delta w_{2}$ can be obtained as follows:

$\delta u_{0}:$

$$
\begin{aligned}
& h^{t} \rho^{t} C^{2} u_{0, x x}+2 h^{t} \rho^{t} C u_{0, x t}+\rho^{t} C^{2} I_{2}^{t} u_{2, x x}+2 \rho^{t} I_{2}^{t} C u_{2, x t}-\frac{1}{2} Q_{66}^{t} I_{2}^{t} v_{2, x y}-Q_{11}^{t} I_{2}^{t} u_{2, x x}-h^{t} Q_{13}^{t} w_{1, x}-\frac{1}{2} h^{t} Q_{66}^{t} v_{0, x y} \\
& -h^{t} Q_{12}^{t} v_{0, x y}+\rho^{t} I_{2}^{t} u_{2, t t}-\frac{1}{2} Q_{66}^{t} I_{2}^{t} u_{2, y y}-Q_{12}^{t} I_{2}^{t} v_{2, x y}+h^{t} \rho^{t} u_{0, t t}-\frac{1}{2} h^{t} Q_{66}^{t} u_{0, y y}-h^{t} Q_{11}^{t} u_{0, x x}+h^{c} \rho^{c} C^{2} u_{0, x x} \\
& +2 h^{c} \rho^{c} C u_{0, x t}+\rho^{c} C^{2} I_{2}^{c} u_{2, x x}+2 \rho^{c} I_{2}^{c} C u_{2, x t}-\frac{1}{2} Q_{66}^{c} I_{2}^{c} v_{2, x y}-Q_{11}^{c} I_{2}^{c} u_{2, x x}-h^{c} Q_{13}^{c} w_{1, x}-\frac{1}{2} h^{c} Q_{66}^{c} v_{0, x y}-h^{c} Q_{12}^{c} v_{0, x y} \\
& +\rho^{c} I_{2}^{c} u_{2, t t}-\frac{1}{2} Q_{66}^{c} I_{2}^{c} u_{2, y y}-Q_{12}^{c} I_{2}^{c} v_{2, x y}+h^{c} \rho^{c} u_{0, t t}-\frac{1}{2} h^{c} Q_{66}^{c} u_{0, y y}-h^{c} Q_{11}^{c} u_{0, x x}+h^{b} \rho^{b} C^{2} u_{0, x x}+2 h^{b} \rho^{b} C u_{0, x t} \\
& +\rho^{b} C^{2} I_{2}^{b} u_{2, x x}+2 \rho^{b} I_{2}^{b} C u_{2, x t}-\frac{1}{2} Q_{66}^{b} I_{2}^{b} v_{2, x y}-Q_{11}^{b} I_{2}^{b} u_{2, x x}-h^{b} Q_{13}^{b} w_{1, x}-\frac{1}{2} h^{b} Q_{66}^{b} v_{0, x y}-h^{b} Q_{12}^{b} v_{0, x y}+\rho^{b} I_{2}^{b} u_{2, t t} \\
& -\frac{1}{2} Q_{66}^{b} I_{2}^{b} u_{2, y y}-Q_{12}^{b} I_{2}^{b} v_{2, x y}+h^{b} \rho^{b} u_{0, t t}-\frac{1}{2} h^{b} Q_{66}^{b} u_{0, y y}-h^{b} Q_{11}^{b} u_{0, x x}=0,
\end{aligned}
$$

$\delta u_{1}$ :

$$
\begin{aligned}
& \frac{1}{2} h^{t} Q_{55}^{t} u_{1}+2 \rho^{t} I_{2}^{t} C u_{1, x t}+\rho^{t} C^{2} I_{2}^{t} u_{1, x x}+\rho^{t} C^{2} I_{4}^{t} u_{3, x x}+2 \rho^{t} I_{4}^{t} C u_{3, x t}-Q_{12}^{t} I_{4}^{t} v_{3, x y}-\frac{1}{2} Q_{66}^{t} I_{4}^{t} u_{3, y y}-\frac{1}{2} Q_{66}^{t} I_{2}^{t} v_{1, x y} \\
& -Q_{12}^{t} I_{2}^{t} v_{1, x y}+\rho^{t} I_{4}^{t} u_{3, t t}+\frac{3}{2} Q_{55}^{t} I_{2}^{t} u_{3}-Q_{11}^{t} I_{2}^{t} u_{1, x x}+\rho^{t} I_{2}^{t} u_{1, t t}+\frac{1}{2} h^{t} Q_{55}^{t} w_{0, x}-Q_{11}^{t} I_{4}^{t} u_{3, x x}-\frac{1}{2} Q_{66}^{t} I_{4}^{t} v_{3, x y}+\frac{1}{2} Q_{55}^{t} I_{2}^{t} w_{2, x} \\
& -2 Q_{13}^{t} I_{2}^{t} w_{2, x}-\frac{1}{2} Q_{66}^{t} I_{2}^{t} u_{1, y y}+\frac{1}{2} h^{c} Q_{55}^{c} u_{1}+2 \rho^{c} I_{2}^{c} C u_{1, x t}+\rho^{c} C^{2} I_{2}^{c} u_{1, x x}+\rho^{c} C^{2} I_{4}^{c} u_{3, x x}+2 \rho^{c} I_{4}^{c} C u_{3, x t}-Q_{12}^{c} I_{4}^{c} v_{3, x y} \\
& -\frac{1}{2} Q_{66}^{c} I_{4}^{c} u_{3, y y}-\frac{1}{2} Q_{66}^{c} I_{2}^{c} v_{1, x y}-Q_{12}^{c} I_{2}^{c} v_{1, x y}+\rho^{c} I_{4}^{c} u_{3, t t}+\frac{3}{2} Q_{55}^{c} I_{2}^{c} u_{3}-Q_{11}^{c} I_{2}^{c} u_{1, x x}+\rho^{c} I_{2}^{c} u_{1, t t}+\frac{1}{2} h^{c} Q_{55}^{c} w_{0, x} \\
& -Q_{11}^{c} I_{4}^{c} u_{3, x x}-\frac{1}{2} Q_{66}^{c} I_{4}^{c} v_{3, x y}+\frac{1}{2} Q_{55}^{c} I_{2}^{c} w_{2, x}-2 Q_{13}^{c} I_{2}^{c} w_{2, x}-\frac{1}{2} Q_{66}^{c} I_{2}^{c} u_{1, x y}+\frac{1}{2} h^{b} Q_{55}^{b} u_{1}+2 \rho^{b} I_{2}^{b} C u_{1, x t}+\rho^{b} C^{2} I_{2}^{b} u_{1, x x} \\
& +\rho^{b} C^{2} I_{4}^{b} u_{3, x x}+2 \rho^{b} I_{4}^{b} C u_{3, x t}-Q_{12}^{b} I_{4}^{b} v_{3, x y}-\frac{1}{2} Q_{66}^{b} I_{4}^{b} u_{3, y y}-\frac{1}{2} Q_{66}^{b} I_{2}^{b} v_{1, x y}-Q_{12}^{b} I_{2}^{b} v_{1, x y}+\rho^{b} I_{4}^{b} u_{3, t t}+\frac{3}{2} Q_{55}^{b} I_{2}^{b} u_{3} \\
& -Q_{11}^{b} I_{2}^{b} u_{1, x x}+\rho^{b} I_{2}^{b} u_{1, t t}+\frac{1}{2} h^{b} Q_{55}^{b} w_{0, x}-Q_{11}^{b} I_{4}^{b} u_{3, x x}-\frac{1}{2} Q_{66}^{b} I_{4}^{b} v_{3, x y}+\frac{1}{2} Q_{55}^{b} I_{2}^{b} w_{2, x}-2 Q_{13}^{b} I_{2}^{b} w_{2, x}-\frac{1}{2} Q_{66}^{b} I_{2}^{b} u_{1, y y}=0,
\end{aligned}
$$

$\delta u_{2}:$

$$
\begin{aligned}
& \rho^{t} C^{2} I_{4}^{t} u_{2, x x}+2 \rho^{t} I_{4}^{t} C u_{2, x t}+\rho^{t} C^{2} I_{2}^{t} u_{0, x x}+2 \rho^{t} I_{2}^{t} C u_{0, x t}-\frac{1}{2} Q_{66}^{t} I_{2}^{t} u_{0, y y}-Q_{12}^{t} I_{2}^{t} v_{0, x y}-Q_{12}^{t} I_{4}^{t} v_{2, x y}-\frac{1}{2} Q_{66}^{t} I_{4}^{t} v_{2, x y} \\
& -Q_{11}^{t} I_{2}^{t} u_{0, x x}-Q_{13}^{t} I_{2}^{t} w_{1, x}+Q_{55}^{t} I_{2}^{t} w_{1, x}-\frac{1}{2} Q_{66}^{t} I_{2}^{t} v_{0, x y}+\rho^{t} I_{2}^{t} u_{0, t t}+2 Q_{55}^{t} I_{2}^{t} u_{2}-\frac{1}{2} Q_{66}^{t} I_{4}^{t} u_{2, y y}-Q_{11}^{t} I_{4}^{t} u_{2, x x}+\rho^{t} I_{4}^{t} u_{2, t t} \\
& +\rho^{c} C^{2} I_{4}^{c} u_{2, x x}+2 \rho^{c} I_{4}^{c} C u_{2, x t}+\rho^{c} C^{2} I_{2}^{c} u_{0, x x}+2 \rho^{c} I_{2}^{c} C u_{0, x t}-\frac{1}{2} Q_{66}^{c} I_{2}^{c} u_{0, y y}-Q_{12}^{c} I_{2}^{c} v_{0, x y}-Q_{12}^{c} I_{4}^{c} v_{2, x y}-\frac{1}{2} Q_{66}^{c} I_{4}^{c} v_{2, x y} \\
& -Q_{11}^{c} I_{2}^{c} u_{0, x x}-Q_{13}^{c} I_{2}^{c} w_{1, x}+Q_{55}^{c} I_{2}^{c} w_{1, x}-\frac{1}{2} Q_{66}^{c} I_{2}^{c} v_{0, x y}+\rho^{c} I_{2}^{c} u_{0, t t}+2 Q_{55}^{c} I_{2}^{c} u_{2}-\frac{1}{2} Q_{66}^{c} I_{4}^{c} u_{2, y y}-Q_{11}^{c} I_{4}^{c} u_{2, x x}+\rho^{c} I_{4}^{c} u_{2, t t} \\
& +\rho^{b} C^{2} I_{4}^{b} u_{2, x x}+2 \rho^{b} I_{4}^{b} C u_{2, x t}+\rho^{b} C^{2} I_{2}^{b} u_{0, x x}+2 \rho^{b} I_{2}^{b} C u_{0, x t}-\frac{1}{2} Q_{66}^{b} I_{2}^{b} u_{0, y y}-Q_{12}^{b} I_{2}^{b} v_{0, x y}-Q_{12}^{b} I_{4}^{b} v_{2, x y}-\frac{1}{2} Q_{66}^{b} I_{4}^{b} v_{2, x y} \\
& -Q_{11}^{b} I_{2}^{b} u_{0, x x}-Q_{13}^{b} I_{2}^{b} w_{1, x}+Q_{55}^{b} I_{2}^{b} w_{1, x}-\frac{1}{2} Q_{66}^{b} I_{2}^{b} v_{0, x y}+\rho^{b} I_{2}^{b} u_{0, t t}+2 Q_{55}^{b} I_{2}^{b} u_{2}-\frac{1}{2} Q_{66}^{b} I_{4}^{b} u_{2, y y}-Q_{11}^{b} I_{4}^{b} u_{2, x x}+\rho^{b} I_{4}^{b} u_{2, t t}=0,
\end{aligned}
$$

44 
$\delta u_{3}:$

$2 \rho^{t} I_{6}^{t} C u_{3, x t}+\rho^{t} C^{2} I_{4}^{t} u_{1, x x}+2 \rho^{t} I_{4}^{t} C u_{1, x t}-\frac{1}{2} Q_{66}^{t} I_{4}^{t} u_{1, y y}-Q_{12}^{t} I_{4}^{t} v_{1, x y}+\rho^{t} I_{4}^{t} u_{1, t t}+\frac{3}{2} Q_{55}^{t} I_{2}^{t} u_{1}-\frac{1}{2} Q_{66}^{t} I_{6}^{t} u_{3, y y}-Q_{11}^{t} I_{6}^{t} u_{3, x x}$ $-2.0 Q_{13}^{t} I_{4}^{t} w_{2, x}-\frac{1}{2} Q_{66}^{t} I_{6}^{t} v_{3, x y}-Q_{11}^{t} I_{4}^{t} u_{1, x x}-Q_{12}^{t} I_{6}^{t} v_{3, x y}+\frac{3}{2} Q_{55}^{t} I_{2}^{t} w_{0, x}-\frac{1}{2} Q_{66}^{t} I_{4}^{t} v_{1, x y}+\frac{3}{2} Q_{55}^{t} I_{4}^{t} w_{2, x}+5 Q_{55}^{t} I_{4}^{t} u_{3}$ $+2 \rho^{t} C^{2} I_{6}^{t} u_{3, x x}+\rho^{t} I_{6}^{t} u_{3, t t}+2 \rho^{c} I_{6}^{c} C u_{3, x t}+\rho^{c} C^{2} I_{4}^{c} u_{1, x x}+2 \rho^{c} I_{4}^{c} C u_{1, x t}-\frac{1}{2} Q_{66}^{c} I_{4}^{c} u_{1, y y}-Q_{12}^{c} I_{4}^{c} v_{1, x y}+\rho^{c} I_{4}^{c} u_{1, t t}$ $+\frac{3}{2} Q_{55}^{c} I_{2}^{c} u_{1}-\frac{1}{2} Q_{66}^{c} I_{6}^{c} u_{3, y y}-Q_{11}^{c} I_{6}^{c} u_{3, x x}-2.0 Q_{13}^{c} I_{4}^{c} w_{2, x}-\frac{1}{2} Q_{66}^{c} I_{6}^{c} v_{3, x y}-Q_{11}^{c} I_{4}^{c} u_{1, x x}-Q_{12}^{c} I_{6}^{c} v_{3, x y}+\frac{3}{2} Q_{55}^{c} I_{2}^{c} w_{0, x}$ $-\frac{1}{2} Q_{66}^{c} I_{4}^{c} v_{1, x y}+\frac{3}{2} Q_{55}^{c} I_{4}^{c} w_{2, x}+5 Q_{55}^{c} I_{4}^{c} u_{3}+2 \rho^{c} C^{2} I_{6}^{c} u_{3, x x}+\rho^{c} I_{6}^{c} u_{3, t t}+2 \rho^{b} I_{6}^{b} C u_{3, x t}+\rho^{b} C^{2} I_{4}^{b} u_{1, x x}+2 \rho^{b} I_{4}^{b} C u_{1, x t}$ $-\frac{1}{2} Q_{66}^{b} I_{4}^{b} u_{1, y y}-Q_{12}^{b} I_{4}^{b} v_{1, x y}+\rho^{b} I_{4}^{b} u_{1, t t}+\frac{3}{2} Q_{55}^{b} I_{2}^{b} u_{1}-\frac{1}{2} Q_{66}^{b} I_{6}^{b} u_{3, y y}-Q_{11}^{b} I_{6}^{b} u_{3, x x}-2.0 Q_{13}^{b} I_{4}^{b} w_{2, x}-\frac{1}{2} Q_{66}^{b} I_{6}^{b} v_{3, x y}$ $-Q_{11}^{b} I_{4}^{b} u_{1, x x}-Q_{12}^{b} I_{6}^{b} v_{3, x y}+\frac{3}{2} Q_{55}^{b} I_{2}^{b} w_{0, x}-\frac{1}{2} Q_{66}^{b} I_{4}^{b} v_{1, x y}+\frac{3}{2} Q_{55}^{b} I_{4}^{b} w_{2, x}+5 Q_{55}^{b} I_{4}^{b} u_{3}+2 \rho^{b} C^{2} I_{6}^{b} u_{3, x x}+\rho^{b} I_{6}^{b} u_{3, t t}=0$,

$\delta v_{0}:$

$h^{t} \rho^{t} C^{2} v_{0, x x}+2 h^{t} \rho^{t} C v_{0, x t}+2 \rho^{t} I_{2}^{t} C v_{2, x t}+\rho^{t} C^{2} I_{2}^{t} v_{2, x x}-Q_{12}^{t} I_{2}^{t} u_{2, x y}+\rho^{t} I_{2}^{t} v_{2, t t}-\frac{1}{2} Q_{66}^{t} I_{2}^{t} v_{2, x x}-h^{t} Q_{23}^{t} w_{1, y}-\frac{1}{2} h^{t} Q_{66}^{t} u_{0, x y}$ $-h^{t} Q_{12}^{t} u_{0, x y}-\frac{1}{2} Q_{66}^{t} I_{2}^{t} u_{2, x y}-Q_{22}^{t} I_{2}^{t} v_{2, y y}+h^{t} \rho^{t} v_{0, t t}-\frac{1}{2} h^{t} Q_{66}^{t} v_{0, x x}-h^{t} Q_{22}^{t} v_{0, y y}+h^{c} \rho^{c} C^{2} v_{0, x x}+2 h^{c} \rho^{c} C v_{0, x t}+2 \rho^{c} I_{2}^{c} C v_{2, x t}$ $+\rho^{c} C^{2} I_{2}^{c} v_{2, x x}-Q_{12}^{c} I_{2}^{c} u_{2, x y}+\rho^{c} I_{2}^{c} v_{2, t t}-\frac{1}{2} Q_{66}^{c} I_{2}^{c} v_{2, x x}-h^{c} Q_{23}^{c} w_{1, y}-\frac{1}{2} h^{c} Q_{66}^{c} u_{0, x y}-h^{c} Q_{12}^{c} u_{0, x y}-\frac{1}{2} Q_{66}^{c} I_{2}^{c} u_{2, x y}-Q_{22}^{c} I_{2}^{c} v_{2, y y}$ $+h^{c} \rho^{c} v_{0, t t}-\frac{1}{2} h^{c} Q_{66}^{c} v_{0, x x}-h^{c} Q_{22}^{c} v_{0, y y}+h^{b} \rho^{b} C^{2} v_{0, x x}+2 h^{b} \rho^{b} C v_{0, x t}+2 \rho^{b} I_{2}^{b} C v_{2, x t}+\rho^{b} C^{2} I_{2}^{b} v_{2, x x}-Q_{12}^{b} I_{2}^{b} u_{2, x y}+\rho^{b} I_{2}^{b} v_{2, t t}$ $-\frac{1}{2} Q_{66}^{b} I_{2}^{b} v_{2, x x}-h^{b} Q_{23}^{b} w_{1, y}-\frac{1}{2} h^{b} Q_{66}^{b} u_{0, x y}-h^{b} Q_{12}^{b} u_{0, x y}-\frac{1}{2} Q_{66}^{b} I_{2}^{b} u_{2, x y}-Q_{22}^{b} I_{2}^{b} v_{2, y y}+h^{b} \rho^{b} v_{0, t t}-\frac{1}{2} h^{b} Q_{66}^{b} v_{0, x x}-h^{b} Q_{22}^{b} v_{0, y y}=0$,

$\delta v_{1}:$

$\frac{1}{2} h^{t} Q_{44}^{t} v_{1}+2 \rho^{t} C^{2} I_{2}^{t} v_{1, x x}+2 \rho^{t} I_{2}^{t} C v_{1, x t}+2 \rho^{t} I_{4}^{t} C v_{3, x t}+\rho^{t} C^{2} I_{4}^{t} v_{3, x x}-Q_{22}^{t} I_{4}^{t} v_{3, y y}$ $-Q_{12}^{t} I_{4}^{t} u_{3, x y}-\frac{1}{2} Q_{66}^{t} I_{2}^{t} u_{1, x y}+\rho^{t} I_{4}^{t} v_{3, t t}-Q_{12}^{t} I_{2}^{t} u_{1, x y}+\frac{1}{2} Q_{44}^{t} I_{2}^{t} w_{2, y}+\frac{3}{2} Q_{44}^{t} I_{2}^{t} v_{3}+\frac{1}{2} h^{t} Q_{44}^{t} w_{0, y}$ $-\frac{1}{2} Q_{66}^{t} I_{4}^{t} v_{3, x x}-\frac{1}{2} Q_{66}^{t} I_{4}^{t} u_{3, x y}-2.0 Q_{23}^{t} I_{2}^{t} w_{2, y}+\rho^{t} I_{2}^{t} v_{1, t t}-Q_{22}^{t} I_{2}^{t} v_{1, y y}-\frac{1}{2} Q_{66}^{t} I_{2}^{t} v_{1, x x}+\frac{1}{2} h^{c} Q_{44}^{c} v_{1}$ $+2 \rho^{c} C^{2} I_{2}^{c} v_{1, x x}+2 \rho^{c} I_{2}^{c} C v_{1, x t}+2 \rho^{c} I_{4}^{c} C v_{3, x t}+\rho^{c} C^{2} I_{4}^{c} v_{3, x x}-Q_{22}^{c} I_{4}^{c} v_{3, y y}-Q_{12}^{c} I_{4}^{c} u_{3, x y}$ $-\frac{1}{2} Q_{66}^{c} I_{2}^{c} u_{1, x y}+\rho^{c} I_{4}^{c} v_{3, t t}-Q_{12}^{c} I_{2}^{c} u_{1, x y}+\frac{1}{2} Q_{44}^{c} I_{2}^{c} w_{2, y}+\frac{3}{2} Q_{44}^{c} I_{2}^{c} v_{3}+\frac{1}{2} h^{c} Q_{44}^{c} w_{0, y}-\frac{1}{2} Q_{66}^{c} I_{4}^{c} v_{3, x x}$ $-\frac{1}{2} Q_{66}^{c} I_{4}^{c} u_{3, x y}-2.0 Q_{23}^{c} I_{2}^{c} w_{2, y}+\rho^{c} I_{2}^{c} v_{1, t t}-Q_{22}^{c} I_{2}^{c} v_{1, y y}-\frac{1}{2} Q_{66}^{c} I_{2}^{c} v_{1, x x}+\frac{1}{2} h^{b} Q_{44}^{b} v_{1}+2 \rho^{b} C^{2} I_{2}^{b} v_{1, x x}$ $+2 \rho^{b} I_{2}^{b} C v_{1, x t}+2 \rho^{b} I_{4}^{b} C v_{3, x t}+\rho^{b} C^{2} I_{4}^{b} v_{3, x x}-Q_{22}^{b} I_{4}^{b} v_{3, y y}-Q_{12}^{b} I_{4}^{b} u_{3, x y}-\frac{1}{2} Q_{66}^{b} I_{2}^{b} u_{1, x y}+\rho^{b} I_{4}^{b} v_{3, t t}$ $-Q_{12}^{b} I_{2}^{b} u_{1, x y}+\frac{1}{2} Q_{44}^{b} I_{2}^{b} w_{2, y}+\frac{3}{2} Q_{44}^{b} I_{2}^{b} v_{3}+\frac{1}{2} h^{b} Q_{44}^{b} w_{0, y}-\frac{1}{2} Q_{66}^{b} I_{4}^{b} v_{3, x x}-\frac{1}{2} Q_{66}^{b} I_{4}^{b} u_{3, x y}-2.0 Q_{23}^{b} I_{2}^{b} w_{2, y}$ $+\rho^{b} I_{2}^{b} v_{1, t t}-Q_{22}^{b} I_{2}^{b} v_{1, y y}-\frac{1}{2} Q_{66}^{b} I_{2}^{b} v_{1, x x}=0$, 
$\delta v_{2}:$

$2 \rho^{t} I_{4}^{t} C v_{2, x t}+\rho^{t} C^{2} I_{4}^{t} v_{2, x x}+2 \rho^{t} I_{2}^{t} C v_{0, x t}+\rho^{t} C^{2} I_{2}^{t} v_{0, x x}-Q_{12}^{t} I_{4}^{t} u_{2, x y}-\frac{1}{2} Q_{66}^{t} I_{4}^{t} u_{2, x y}+\rho^{t} I_{2}^{t} v_{0, t t}-\frac{1}{2} Q_{66}^{t} I_{2}^{t} u_{0, x y}$ $-\frac{1}{2} Q_{66}^{t} I_{2}^{t} v_{0, x x}-Q_{23}^{t} I_{2}^{t} w_{1, y}+\rho^{t} I_{4}^{t} v_{2, t t}+Q_{44}^{t} I_{2}^{t} w_{1, y}-Q_{22}^{t} I_{2}^{t} v_{0, y y}-Q_{12}^{t} I_{2}^{t} u_{0, x y}+2 Q_{44}^{t} I_{2}^{t} v_{2}-Q_{22}^{t} I_{4}^{t} v_{2, y y}$ $-\frac{1}{2} Q_{66}^{t} I_{4}^{t} v_{2, x x}+2 \rho^{c} I_{4}^{c} C v_{2, x t}+\rho^{c} C^{2} I_{4}^{c} v_{2, x x}+2 \rho^{c} I_{2}^{c} C v_{0, x t}+\rho^{c} C^{2} I_{2}^{c} v_{0, x x}-Q_{12}^{c} I_{4}^{c} u_{2, x y}-\frac{1}{2} Q_{66}^{c} I_{4}^{c} u_{2, x y}$ $+\rho^{c} I_{2}^{c} v_{0, t t}-\frac{1}{2} Q_{66}^{c} I_{2}^{c} u_{0, x y}-\frac{1}{2} Q_{66}^{c} I_{2}^{c} v_{0, x x}-Q_{23}^{c} I_{2}^{c} w_{1, y}+\rho^{c} I_{4}^{c} v_{2, t t}+Q_{44}^{c} I_{2}^{c} w_{1, y}-Q_{22}^{c} I_{2}^{c} v_{0, y y}-Q_{12}^{c} I_{2}^{c} u_{0, x y}+2 Q_{44}^{c} I_{2}^{c} v_{2}$

$-Q_{22}^{c} I_{4}^{c} v_{2, y y}-\frac{1}{2} Q_{66}^{c} I_{4}^{c} v_{2, x x}+2 \rho^{b} I_{4}^{b} C v_{2, x t}+\rho^{b} C^{2} I_{4}^{b} v_{2, x x}+2 \rho^{b} I_{2}^{b} C v_{0, x t}+\rho^{b} C^{2} I_{2}^{b} v_{0, x x}-Q_{12}^{b} I_{4}^{b} u_{2, x y}$ $-\frac{1}{2} Q_{66}^{b} I_{4}^{b} u_{2, x y}+\rho^{b} I_{2}^{b} v_{0, t t}-\frac{1}{2} Q_{66}^{b} I_{2}^{b} u_{0, x y}-\frac{1}{2} Q_{66}^{b} I_{2}^{b} v_{0, x x}-Q_{23}^{b} I_{2}^{b} w_{1, y}+\rho^{b} I_{4}^{b} v_{2, t t}+Q_{44}^{b} I_{2}^{b} w_{1, y}-Q_{22}^{b} I_{2}^{b} v_{0, y y}$ $-Q_{12}^{b} I_{2}^{b} u_{0, x y}+2 Q_{44}^{b} I_{2}^{b} v_{2}-Q_{22}^{b} I_{4}^{b} v_{2, y y}-\frac{1}{2} Q_{66}^{b} I_{4}^{b} v_{2, x x}=0$,

$\delta v_{3}:$

$2 \rho^{t} I_{6}^{t} C v_{3, x t}+2 \rho^{t} C^{2} I_{6}^{t} v_{3, x x}+2 \rho^{t} I_{4}^{t} C v_{1, x t}+\rho^{t} C^{2} I_{4}^{t} v_{1, x x}-Q_{12}^{t} I_{4}^{t} u_{1, x y}-Q_{22}^{t} I_{4}^{t} v_{1, y y}+\rho^{t} I_{4}^{t} v_{1, t t}+\frac{3}{2} Q_{44}^{t} I_{4}^{t} w_{2, y}$ $-2.0 Q_{23}^{t} I_{4}^{t} w_{2, y}-\frac{1}{2} Q_{66}^{t} I_{6}^{t} v_{3, x x}-\frac{1}{2} Q_{66}^{t} I_{6}^{t} u_{3, x y}-\frac{1}{2} Q_{66}^{t} I_{4}^{t} u_{1, x y}-Q_{12}^{t} I_{6}^{t} u_{3, x y}-\frac{1}{2} Q_{66}^{t} I_{4}^{t} v_{1, x x}+\frac{3}{2} Q_{44}^{t} I_{2}^{t} v_{1}+\frac{3}{2} Q_{44}^{t} I_{2}^{t} w_{0, y}$ $+5 Q_{44}^{t} I_{4}^{t} v_{3}+\rho^{t} I_{6}^{t} v_{3, t t}-Q_{22}^{t} I_{6}^{t} v_{3, y y}+2 \rho^{c} I_{6}^{c} C v_{3, x t}+2 \rho^{c} C^{2} I_{6}^{c} v_{3, x x}+2 \rho^{c} I_{4}^{c} C v_{1, x t}+\rho^{c} C^{2} I_{4}^{c} v_{1, x x}-Q_{12}^{c} I_{4}^{c} u_{1, x y}$ $-Q_{22}^{c} I_{4}^{c} v_{1, y y}+\rho^{c} I_{4}^{c} v_{1, t}+\frac{3}{2} Q_{44}^{c} I_{4}^{c} w_{2, y}-2.0 Q_{23}^{c} I_{4}^{c} w_{2, y}-\frac{1}{2} Q_{66}^{c} I_{6}^{c} v_{3, x x}-\frac{1}{2} Q_{66}^{c} I_{6}^{c} u_{3, x y}-\frac{1}{2} Q_{66}^{c} I_{4}^{c} u_{1, x y}-Q_{12}^{c} I_{6}^{c} u_{3, x y}$ $-\frac{1}{2} Q_{66}^{c} I_{4}^{c} v_{1, x x}+\frac{3}{2} Q_{44}^{c} I_{2}^{c} v_{1}+\frac{3}{2} Q_{44}^{c} I_{2}^{c} w_{0, y}+5 Q_{44}^{c} I_{4}^{c} v_{3}+\rho^{c} I_{6}^{c} v_{3, t}-Q_{22}^{c} I_{6}^{c} v_{3, y y}+2 \rho^{b} I_{6}^{b} C v_{3, x t}+2 \rho^{b} C^{2} I_{6}^{b} v_{3, x x}$ $+2 \rho^{b} I_{4}^{b} C v_{1, x t}+\rho^{b} C^{2} I_{4}^{b} v_{1, x x}-Q_{12}^{b} I_{4}^{b} u_{1, x y}-Q_{22}^{b} I_{4}^{b} v_{1, y y}+\rho^{b} I_{4}^{b} v_{1, t t}+\frac{3}{2} Q_{44}^{b} I_{4}^{b} w_{2, y}-2.0 Q_{23}^{b} I_{4}^{b} w_{2, y}-\frac{1}{2} Q_{66}^{b} I_{6}^{b} v_{3, x x}$ $-\frac{1}{2} Q_{66}^{b} I_{6}^{b} u_{3, x y}-\frac{1}{2} Q_{66}^{b} I_{4}^{b} u_{1, x y}-Q_{12}^{b} I_{6}^{b} u_{3, x y}-\frac{1}{2} Q_{66}^{b} I_{4}^{b} v_{1, x x}+\frac{3}{2} Q_{44}^{b} I_{2}^{b} v_{1}+\frac{3}{2} Q_{44}^{b} I_{2}^{b} w_{0, y}+5 Q_{44}^{b} I_{4}^{b} v_{3}+\rho^{b} I_{6}^{b} v_{3, t t}$ $-Q_{22}^{b} I_{6}^{b} v_{3, y y}=0$,

$\delta w_{0}:$

$h^{t} \rho^{t} C^{2} w_{0, x x}+2 h^{t} \rho^{t} C w_{0, x t}+2 \rho^{t} I_{2}^{t} C w_{2, x t}+\rho^{t} C^{2} I_{2}^{t} w_{2, x x}+\rho^{t} I_{2}^{t} w_{2, t t}-\frac{1}{2} Q_{44}^{t} I_{2}^{t} w_{2, y y}-\frac{1}{2} h^{t} Q_{44}^{t} v_{1, y}-\frac{1}{2} h^{t} Q_{55}^{t} u_{1, x}$ $-\frac{1}{2} Q_{55}^{t} I_{2}^{t} w_{2, x x}-\frac{3}{2} Q_{55}^{t} I_{2}^{t} u_{3, x}-\frac{3}{2} Q_{44}^{t} I_{2}^{t} v_{3, y}+h^{t} \rho^{t} w_{0, t t}-\frac{1}{2} h^{t} Q_{44}^{t} w_{0, y y}-\frac{1}{2} h^{t} Q_{55}^{t} w_{0, x x}+h^{c} \rho^{c} C^{2} w_{0, x x}+2 h^{c} \rho^{c} C w_{0, x t}$ $+2 \rho^{c} I_{2}^{c} C w_{2, x t}+\rho^{c} C^{2} I_{2}^{c} w_{2, x x}+\rho^{c} I_{2}^{c} w_{2, t t}-\frac{1}{2} Q_{44}^{c} I_{2}^{c} w_{2, y y}-\frac{1}{2} h^{c} Q_{44}^{c} v_{1, y}-\frac{1}{2} h^{c} Q_{55}^{c} u_{1, x}-\frac{1}{2} Q_{55}^{c} I_{2}^{c} w_{2, x x}-\frac{3}{2} Q_{55}^{c} I_{2}^{c} u_{3, x}$ $-\frac{3}{2} Q_{44}^{c} I_{2}^{c} v_{3, y}+h^{c} \rho^{c} w_{0, t t}-\frac{1}{2} h^{c} Q_{44}^{c} w_{0, y y}-\frac{1}{2} h^{c} Q_{55}^{c} w_{0, x x}+h^{b} \rho^{b} C^{2} w_{0, x x}+2 h^{b} \rho^{b} C w_{0, x t}+2 \rho^{b} I_{2}^{b} C w_{2, x t}+\rho^{b} C^{2} I_{2}^{b} w_{2, x x}$ $+\rho^{b} I_{2}^{b} w_{2, t}-\frac{1}{2} Q_{44}^{b} I_{2}^{b} w_{2, y y}-\frac{1}{2} h^{b} Q_{44}^{b} v_{1, y}-\frac{1}{2} h^{b} Q_{55}^{b} u_{1, x}-\frac{1}{2} Q_{55}^{b} I_{2}^{b} w_{2, x x}-\frac{3}{2} Q_{55}^{b} I_{2}^{b} u_{3, x}-\frac{3}{2} Q_{44}^{b} I_{2}^{b} v_{3, y}+h^{b} \rho^{b} w_{0, t}$ $-\frac{1}{2} h^{b} Q_{44}^{b} w_{0, y y}-\frac{1}{2} h^{b} Q_{55}^{b} w_{0, x x}-N_{x} h^{t} w_{0, x x}-N_{x} h^{b} w_{0, x x}-N_{x} h^{c} w_{0, x x}=0$, 
$\delta w_{1}:$

$$
\begin{aligned}
& h^{t} Q_{33}^{t} w_{1}+2 \rho^{t} I_{2}^{t} C w_{1, x t}+Q_{23}^{t} I_{2}^{t} v_{2, y}+Q_{13}^{t} I_{2}^{t} u_{2, x}+\rho^{t} I_{2}^{t} w_{1, t t}-Q_{55}^{t} I_{2}^{t} u_{2, x}+h^{t} Q_{23}^{t} v_{0, y}+h^{t} Q_{13}^{t} u_{0, x} \\
& -Q_{44}^{t} I_{2}^{t} v_{2, y}+\rho^{t} C^{2} I_{2}^{t} w_{1, x x}-\frac{1}{2} Q_{55}^{t} I_{2}^{t} w_{1, x x}-\frac{1}{2} Q_{44}^{t} I_{2}^{t} w_{1, y y}+h^{c} Q_{33}^{c} w_{1}+2 \rho^{c} I_{2}^{c} C w_{1, x t}+Q_{23}^{c} I_{2}^{c} v_{2, y} \\
& +Q_{13}^{c} I_{2}^{c} u_{2, x}+\rho^{c} I_{2}^{c} w_{1, t t}-Q_{55}^{c} I_{2}^{c} u_{2, x}+h^{c} Q_{23}^{c} v_{0, y}+h^{c} Q_{13}^{c} u_{0, x}-Q_{44}^{c} I_{2}^{c} v_{2, y}+\rho^{c} C^{2} I_{2}^{c} w_{1, x x}-Q_{55}^{b} I_{2}^{b} u_{2, x} \\
& -\frac{1}{2} Q_{55}^{c} I_{2}^{c} w_{1, x x}-\frac{1}{2} Q_{44}^{c} I_{2}^{c} w_{1, y y}+h^{b} Q_{33}^{b} w_{1}+2 \rho^{b} I_{2}^{b} C w_{1, x t}+Q_{23}^{b} I_{2}^{b} v_{2, y}+Q_{13}^{b} I_{2}^{b} u_{2, x}+\rho^{b} I_{2}^{b} w_{1, t t} \\
& +h^{b} Q_{23}^{b} v_{0, y}+h^{b} Q_{13}^{b} u_{0, x}-Q_{44}^{b} I_{2}^{b} v_{2, y}+\rho^{b} C^{2} I_{2}^{b} w_{1, x x}-\frac{1}{2} Q_{55}^{b} I_{2}^{b} w_{1, x x}-\frac{1}{2} Q_{44}^{b} I_{2}^{b} w_{1, y y}=0,
\end{aligned}
$$

$\delta w_{2}$ :

$2 \rho^{t} I_{4}^{t} C w_{2, x t}+\rho^{t} C^{2} I_{4}^{t} w_{2, x x}+2 \rho^{t} I_{2}^{t} C w_{0, x t}+\rho^{t} C^{2} I_{2}^{t} w_{0, x x}+\rho^{t} I_{2}^{t} w_{0, t t}-\frac{1}{2} Q_{44}^{t} I_{2}^{t} w_{0, y y}-\frac{3}{2} Q_{44}^{t} I_{4}^{t} v_{3, y}-\frac{1}{2} Q_{44}^{t} I_{2}^{t} v_{1, y}$ $+2.0 Q_{23}^{t} I_{4}^{t} v_{3, y}+2.0 Q_{13}^{t} I_{4}^{t} u_{3, x}-\frac{1}{2} Q_{55}^{t} I_{2}^{t} w_{0, x x}-\frac{1}{2} Q_{55}^{t} I_{2}^{t} u_{1, x}+2.0 Q_{13}^{t} I_{2}^{t} u_{1, x}+2.0 Q_{23}^{t} I_{2}^{t} v_{1, y}-\frac{3}{2} Q_{55}^{t} I_{4}^{t} u_{3, x}+4 Q_{33}^{t} I_{2}^{t} w_{2}$ $-\frac{1}{2} Q_{44}^{t} I_{4}^{t} w_{2, y y}+\rho^{t} I_{4}^{t} w_{2, t t}-\frac{1}{2} Q_{55}^{t} I_{4}^{t} w_{2, x x}+2 \rho^{c} I_{4}^{c} C w_{2, x t}+\rho^{c} C^{2} I_{4}^{c} w_{2, x x}+2 \rho^{c} I_{2}^{c} C w_{0, x t}+\rho^{c} C^{2} I_{2}^{c} w_{0, x x}+\rho^{c} I_{2}^{c} w_{0, t t}$ $-\frac{1}{2} Q_{44}^{c} I_{2}^{c} w_{0, y y}-\frac{3}{2} Q_{44}^{c} I_{4}^{c} v_{3, y}-\frac{1}{2} Q_{44}^{c} I_{2}^{c} v_{1, y}+2.0 Q_{23}^{c} I_{4}^{c} v_{3, y}+2.0 Q_{13}^{c} I_{4}^{c} u_{3, x}-\frac{1}{2} Q_{55}^{c} I_{2}^{c} w_{0, x x}-\frac{1}{2} Q_{55}^{c} I_{2}^{c} u_{1, x}+2.0 Q_{13}^{c} I_{2}^{c} u_{1, x}$ $+2.0 Q_{23}^{c} I_{2}^{c} v_{1, y}-\frac{3}{2} Q_{55}^{c} I_{4}^{c} u_{3, x}+4 Q_{33}^{c} I_{2}^{c} w_{2}-\frac{1}{2} Q_{44}^{c} I_{4}^{c} w_{2, y y}+\rho^{c} I_{4}^{c} w_{2, t t}-\frac{1}{2} Q_{55}^{c} I_{4}^{c} w_{2, x x}+2 \rho^{b} I_{4}^{b} C w_{2, x t}+\rho^{b} C^{2} I_{4}^{b} w_{2, x x}$ $+2 \rho^{b} I_{2}^{b} C w_{0, x t}+\rho^{b} C^{2} I_{2}^{b} w_{0, x x}+\rho^{b} I_{2}^{b} w_{0, t t}-\frac{1}{2} Q_{44}^{b} I_{2}^{b} w_{0, y y}-\frac{3}{2} Q_{44}^{b} I_{4}^{b} v_{3, y}-\frac{1}{2} Q_{44}^{b} I_{2}^{b} v_{1, y}+2.0 Q_{23}^{b} I_{4}^{b} v_{3, y}+2.0 Q_{13}^{b} I_{4}^{b} u_{3, x}$ $-\frac{1}{2} Q_{55}^{b} I_{2}^{b} w_{0, x x}-\frac{1}{2} Q_{55}^{b} I_{2}^{b} u_{1, x}+2.0 Q_{13}^{b} I_{2}^{b} u_{1, x}+2.0 Q_{23}^{b} I_{2}^{b} v_{1, y}-\frac{3}{2} Q_{55}^{b} I_{4}^{b} u_{3, x}+4 Q_{33}^{b} I_{2}^{b} w_{2}-\frac{1}{2} Q_{44}^{b} I_{4}^{b} w_{2, y y}+\rho^{b} I_{4}^{b} w_{2, t t}$ $-\frac{1}{2} Q_{55}^{b} I_{4}^{b} w_{2, x x}-N_{x} I_{2}^{t} w_{2, x x}-N_{x} I_{2}^{b} w_{2, x x}-N_{x} I_{2}^{c} w_{2, x x}=0$,

where $I_{i}^{t}, I_{i}^{c}$ and $I_{i}^{b}$ are defined for top, core and bottom layers, respectively, as follows:

$$
I_{2,4,6}^{t}=\int_{+h^{c} / 2}^{+\left(\frac{h^{c}}{2}+h^{t}\right)} z^{2,4,6} d z, \quad I_{2,4,6}^{c}=\int_{-h^{c} / 2}^{h^{c} / 2} z^{2,4,6} d z, \quad I_{2,4,6}^{b}=\int_{-\left(\frac{h^{c}}{2}+h^{b}\right)}^{-h^{c} / 2} z^{2,4,6} d z
$$

\section{Analytical Solution}

The analytical solution of Eqs. (11) exists for the simply-supported axially moving rectangular sandwich plate with composite face sheets. In this approach, the displacements are considered as functions which satisfy at least the various geometric boundary conditions. Based on Navier's procedure, the solution of the displacement variables can be expressed in the following forms [15]: 


$$
\begin{aligned}
& u_{i}(x, y, t)=\sum_{n=1}^{N} \sum_{m=1}^{M} U_{i}^{m n} \cos (\alpha x) \sin (\beta y) \mathrm{e}^{i \Omega t}, \\
& v_{i}(x, y, t)=\sum_{n=1}^{N} \sum_{m=1}^{M} V_{i}^{m n} \sin (\alpha x) \cos (\beta y) \mathrm{e}^{i \Omega t}, \\
& w_{i}(x, y, t)=\sum_{n=1}^{N} \sum_{m=1}^{M} W_{i}^{m n} \sin (\alpha x) \sin (\beta y) \mathrm{e}^{i \Omega t},
\end{aligned}
$$

Substituting above relations into Eqs. (11) lead to final equations as a matrix form:

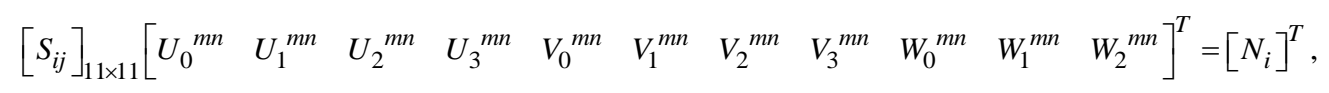

in which, $N_{i}(i=1,2,3)$ is related to external thermal or mechanical loads. It should be noted that in the case of free vibration $N_{i}(i=1,2,3)$ are assumed to be zero. The arrays of matrix $S_{i j}$ are obtained from Eqs. (11) and (13).

\section{Numerical results and discussion}

In this section, effects of various parameters such as volume fraction of CNTs, axially moving speed, aspect ratio and thickness on the vibration characteristics of axially moving sandwich plate with composite face sheets are discussed in details. In the present study, Titanium alloy (Ti-6Al-4V) is considered for the homogeneous core. Poly methyl methacrylate, referred to as PMMA, is selected for the matrix of composite face sheets inside CNTs fibers. The effective material properties of CNTs, Ti-6Al-4V and PMMA are presented in Table 1 and 2. It should be noted that $\eta_{1}=0.137, \eta_{2}=1.022$ and $\eta_{3}=0.715$ for the case of $V_{C N T}^{*}=0.12, \eta_{1}=0.142, \eta_{2}=1.626$ and $\eta_{3}=1.138$ for the case of $V_{C N T}^{*}=0.17$, and $\eta_{1}=0.141, \eta_{2}=1.585$ and $\eta_{3}=1.109$ for the case of $V_{C N T}^{*}=0.28$. Moreover, it's assumed that $G_{12}=G_{13}$ and $G_{23}=1.2 G_{12}$ according to Wang and Shen [22].

Table 1. Mechanical properties of SWCNT with 10 [22].

\begin{tabular}{c|c|c|c|c|c}
\hline $\begin{array}{c}\text { Temperature } \\
(\mathrm{K})\end{array}$ & $E_{11}{ }^{C N T}(T P a)$ & $E_{22}{ }^{C N T}(T P a)$ & $G_{12}{ }^{C N T}(\mathrm{TPa})$ & $v^{C N T}$ & $\rho^{C N T}\left(\mathrm{Kg} / \mathrm{m}^{3}\right)$ \\
\hline 300 & 5.6466 & 7.0800 & 1.9445 & 0.19 & 1400 \\
500 & 5.5308 & 6.9348 & 1.9643 & 0.19 & 1400 \\
700 & 5.4744 & 6.8641 & 1.9644 & 0.19 & 1400 \\
\hline
\end{tabular}

Table 2. Mechanical properties of PMMA and Ti-6Al-4V [22].

\begin{tabular}{c|c|c|c}
\hline Material & $E(\mathrm{GPa})$ & $\rho\left(\mathrm{Kg} / \mathrm{m}^{3}\right)$ & $v$ \\
\hline PMMA & $3.52-0.0034 \mathrm{~T}$ & 1150 & 0.34 \\
Ti-6Al-4V & $122.56\left(1-4.586 \times 10^{-4} \mathrm{~T}\right)$ & 4429 & 0.29 \\
\hline
\end{tabular}

Dimensionless parameters are defined to obtain dimensionless results: 


$$
(\zeta, \eta)=\left(\frac{x}{a}, \frac{y}{b}\right),(U, V, W)=\left(\frac{u}{a}, \frac{v}{b}, \frac{w}{h}\right),(\alpha, \beta, \gamma)=\left(\frac{h}{a}, \frac{h}{b}, \frac{a}{b}\right), C^{*}=C \sqrt{\frac{Q_{11}{ }^{c}}{\rho^{c}}} P^{*}=\frac{N_{x}}{Q_{11}{ }^{c} a} \quad \Omega=\omega a \sqrt{\frac{\rho^{c}}{Q_{11}}}
$$

Fig. 2 illustrates the influence of volume fractions of CNTs on the dimensionless frequencies of axially moving sandwich plate. This figure shows that increasing volume fractions of CNTs leads to increase stiffness of sandwich plate and consequently the frequencies of moving system increase. In addition, it's evident that increasing $V_{C N T}^{*}$ from 0.17 to 0.28 not considerably affected the natural frequencies of moving system, especially at lower aspect ratios. Moreover, it can be observed that the frequencies moving system increase with increasing aspect ratios of sandwich plate.

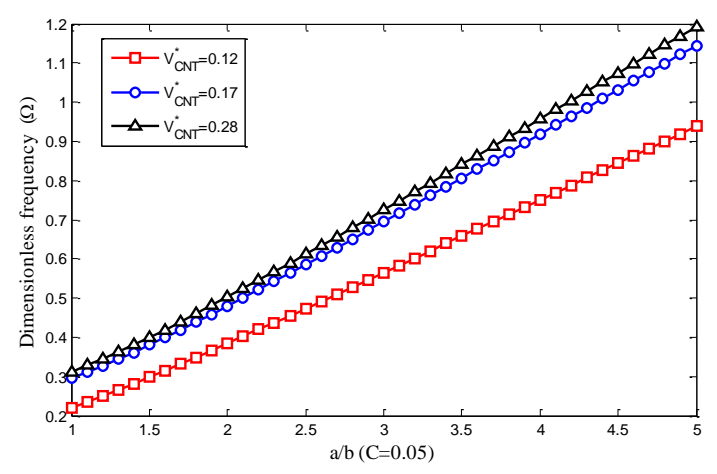

Fig. 2. Dimensionless frequency versus aspect ratio of sandwich plate for different volume fractions of CNTs.

The real part of dimensionless frequency versus dimensionless axially moving speed for different core thickness is depicted in Fig.3. As can be observed, $\operatorname{Im}(\omega)$ diminishes with increasing $C$. These physically proved that the system is stable and the small moving speed does not result in damping behavior. For zero resonance frequency, axially moving sandwich plate becomes unstable due to the divergence via a pitchfork bifurcation and the corresponding moving speed is called the critical speed. Therefore, with increasing moving speed, system stability decreases and became susceptible to buckling. It is obvious that increasing core thickness causes to increase strength of sandwich plate and consequently the frequencies of system increase.

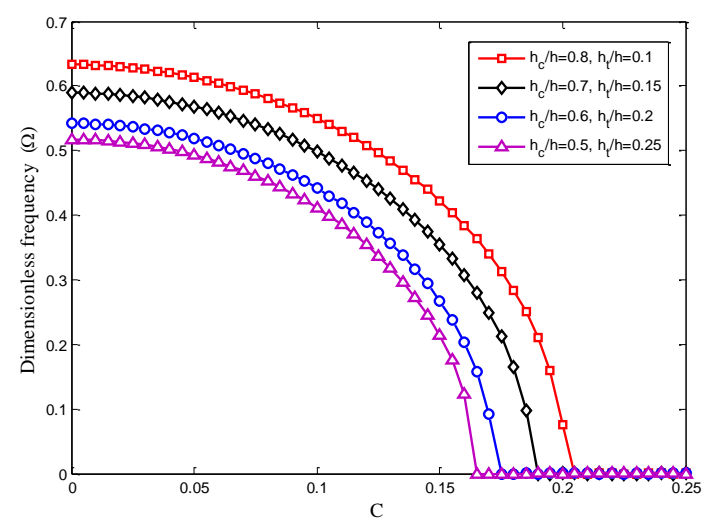

Fig. 3. Dimensionless frequency versus dimensionless moving speed of sandwich plate in different values of core and face sheets thickness. 
The influences of volume fractions of sandwich plate on dimensionless frequencies versus dimensionless thickness parameter are demonstrated in Fig.4. This figure approved that increasing thickness of sandwich plate leads to increase frequencies of moving system. In addition, the effect of CNTs reinforcement is more significant at thicker sandwich plate. Also, it can be found that the frequencies of sandwich plate which is reinforced by 0.17 and 0.28 volume fractions of CNTs are similar. So, in this study $V_{C N T}^{*}=0.17$ is selected for the face sheets of sandwich plate.

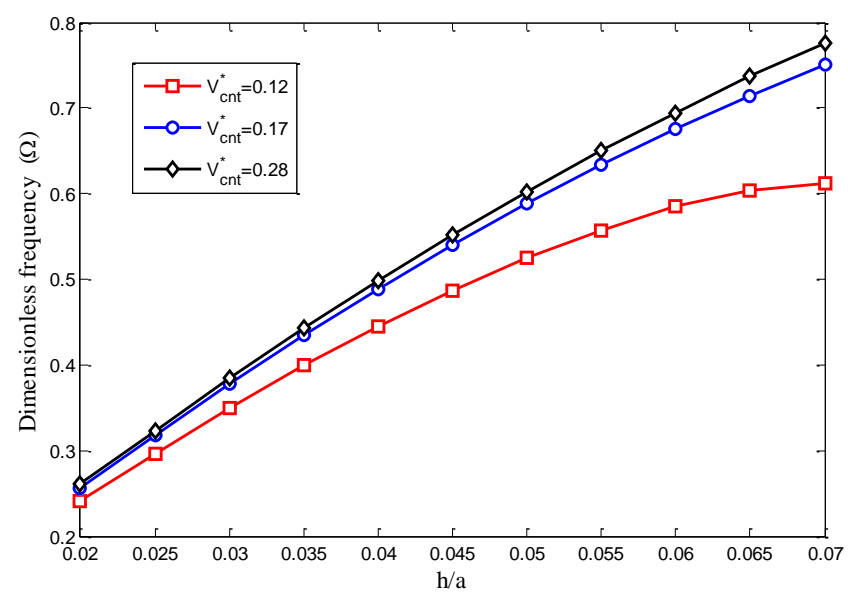

Fig. 4. The influence of CNTs volume fraction on dimensionless frequency versus dimensionless thickness ratio of sandwich plate.

As mentioned ago, SWCNTs is selected as a reinforcement of face sheets of sandwich plate. The mechanical properties of CNTs at different temperatures are adopted from Wang and Shen (2012). Fig. 5 presents the effect of temperature on vibration frequencies of moving sandwich plate. As can be seen, increasing temperature leads to increase the frequencies of moving composite plate, especially at higher thickness of plate.

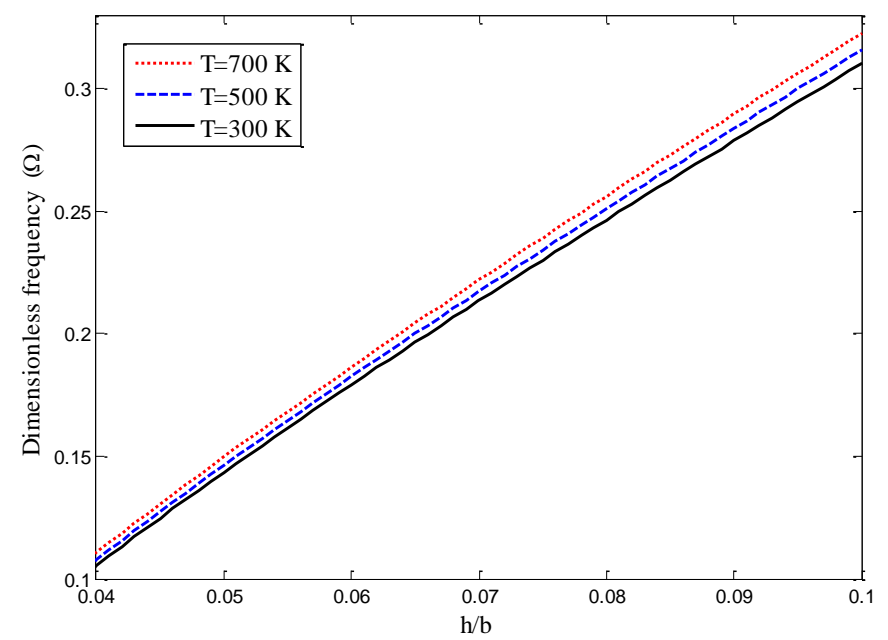

Fig. 5. The effect of temperature on dimensionless frequencies of axially moving sandwich plate versus dimensionless thickness ratio of sandwich plate.

Fig.6 shows the influences of temperature changes and volume fractions on dimensionless frequencies versus dimensionless core thickness parameter, simultaneously. This figure approved 
that volume fractions of CNTs and temperature changes are a significant parameters which are changed frequencies of moving sandwich plate, considerably.

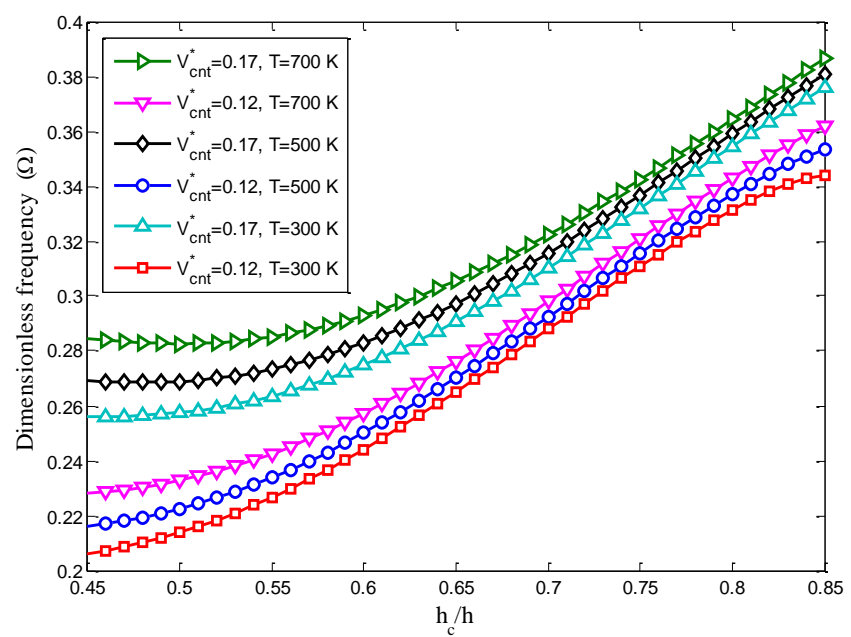

Fig. 6. Dimensionless frequency versus dimensionless core thickness of sandwich plate in different temperature and volume fractions of CNTs.

The effect of moving speed of sandwich plate on dimensionless frequency versus dimensionless aspect ratio is demonstrated in Fig. 7. It can be found from this figure that the values of critical speed in square plate are lower than rectangular plate. Moreover, increasing moving speed leads to increase instability of sandwich plate and consequently the frequencies decrease.

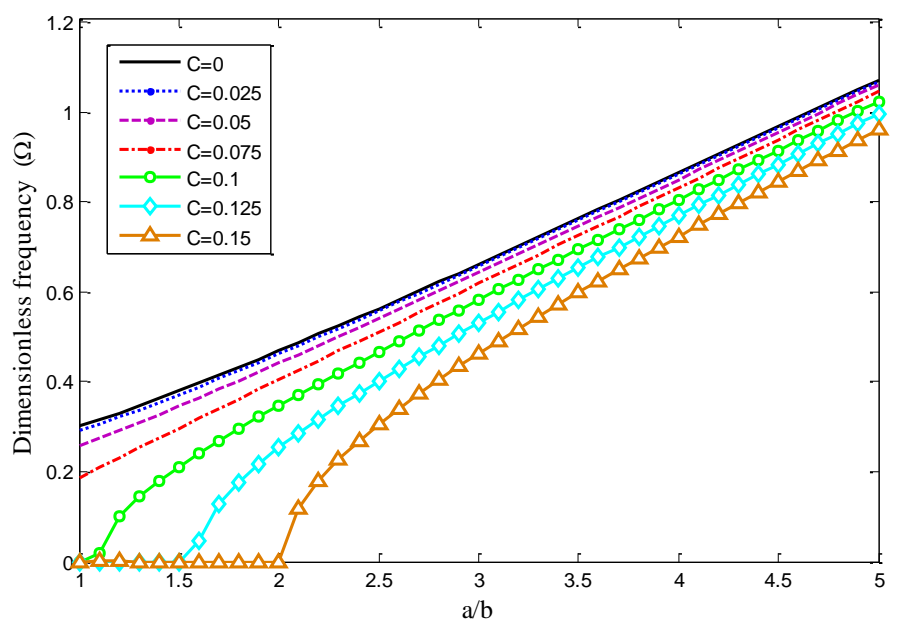

Fig. 7. The effect of moving speed on dimensionless frequency versus aspect ratio of sandwich plate.

Dimensionless frequencies versus dimensionless initial tension in different moving speeds are demonstrated in Fig.8. It's concluded that increasing pre-tension leads to decrease dimensionless frequency of sandwich plate. In addition, the influence of initial tension in axially moving plate with higher moving speeds is more considerable than stationary plates. 


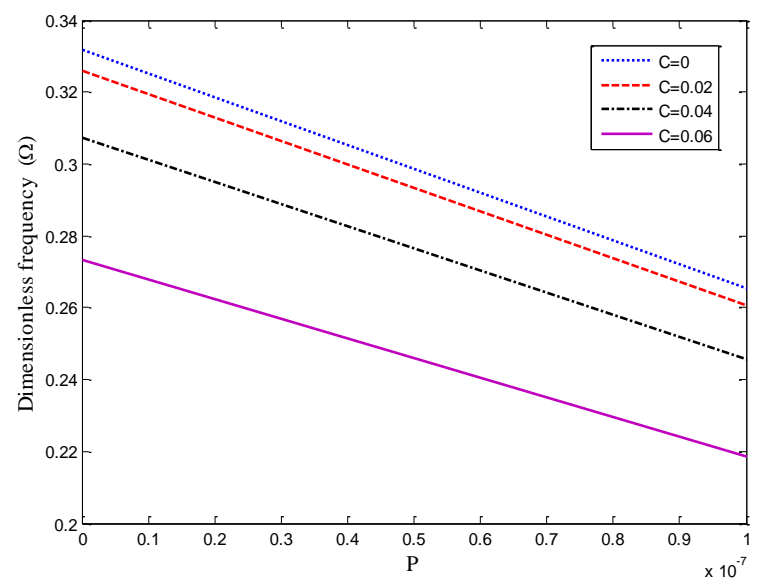

Fig. 8. The effect of moving speed on the dimensionless frequency versus dimensionless initial tension.

Fig.9 illustrates the effect of vibration modes on dimensionless frequencies versus dimensionless moving speed of sandwich plate. It is evident that the critical speed and frequencies of sandwich plate in third mode are higher than the first mode.

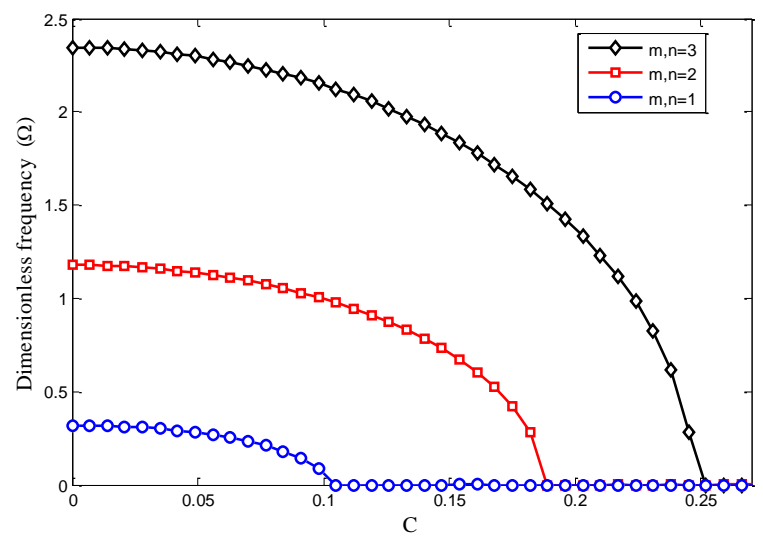

Fig. 9. The effect of vibration modes on the dimensionless frequency versus dimensionless moving speed of sandwich plate.

In order to examine the reliability of the presented method, the results of this method are compared with the work by Wang and Shen (2012). For this purpose, sandwich plate with CNTRC face sheets is considered. Non-dimensional natural frequencies are obtained by $\Omega=\omega a^{2} / h\left(\sqrt{\rho^{c} / E^{c}}\right)$ where $\rho^{c}$ and $E^{c}$ represents mass density and Young's module of core layer at $\mathrm{T}=300 \mathrm{~K}$. As can be seen, there are good agreement between the results of present study and their approach.

Table 3. Comparison between non-dimensional natural frequencies of sandwich plate with CNTRC face sheets $(\mathrm{C}=0, \mathrm{a} / \mathrm{b}=1, \mathrm{~b} / \mathrm{h}=20)$

\begin{tabular}{c|c|c|c|c|c|c}
\hline \multirow{2}{*}{$\mathrm{T}=300 \mathrm{~K}$} & \multicolumn{2}{|c|}{$\frac{h_{c}}{h_{t}}=8$} & \multicolumn{2}{c|}{$\frac{h_{c}}{h_{t}}=6$} & \multicolumn{2}{c}{$\frac{h_{c}}{h_{t}}=4$} \\
\hline$V_{C N T}^{*}$ & 0.17 & 0.28 & 0.17 & 0.28 & 0.17 & 0.28 \\
\hline Present & 4.5577 & 4.5673 & 4.2701 & 4.2710 & 3.7173 & 3.7203 \\
\hline Ref. [22] & 4.5887 & 4.5871 & 4.2642 & 4.2939 & 3.7320 & 3.7378 \\
\hline
\end{tabular}




\section{Conclusion}

Based on HSDT, vibration analysis of axially moving sandwich plate with composite face sheets was developed for the first time. PMMA was selected as a matrix composite face sheets inside CNTs fibers. Extended rule of mixture was utilized to obtain structural properties of composite face sheets. Considering simply supported boundary condition, the motion equations were obtained using Hamilton's principle and solved by analytical solution. It was found that vibrating behavior of moving sandwich plate was strongly dependent on moving speed, so that, with increasing moving speed, system stability decreases and became susceptible to buckling. In addition, increasing small amount in volume fraction of fibers led to increase frequencies of sandwich plate, considerably. Comparison between natural frequencies of this study and the work which was done by Wang and Shen [22] confirmed the accuracy of presented results. The results of this study is hoped to be used in optimum design of aircrafts and military equipment.

\section{Acknowledgements}

The authors are grateful to University of Kashan for supporting this work by Grant No. 574600/24.

\section{References}

[1] Thostenson, E.T., Wei Chou, T., On the elastic properties of carbon nanotube-based composites: modelling and characterization. Journal of Physics D: Applied Physics, 36, 573-582, 2003.

[2] Zhu, P., Lei, Z.X., Liew, K.L., Static and free vibration analyses of carbon nanotube-reinforced composite plates using finite element method with first order shear deformation plate theory. Composite Structures, 94, 1450-1460, 2012.

[3] Lei, Z.X., Liew, K.M., Yu, J.L., Free vibration analysis of functionally graded carbon nanotubereinforced composite plates using the element-free kp-Ritz method in thermal environment. Composite Structures, 106, 128-138, 2013.

[4] Alibeigloo, A., Static analysis of functionally graded carbon nanotube-reinforced composite plate embedded in piezoelectric layers by using theory of elasticity. Composite Structures, 95, 612-622, 2013.

[5] Nayak, A.K., Moy, S.S.J., Shenoi, R.A., Free vibration analysis of composite sandwich plates based on Reddy's higher-order theory, Composite Part B: Engineering, 33, 505-519, 2002.

[6] Ferreira, A.J.M., Roque, C.M.C., Jorge, R.M.N., Kansa, E.J., Radial basis functions collocation and a unified formulation for bending, vibration and buckling analysis of laminated plates, according to variation of Murakami's zigzag theory. European Journal of Mechanics - A/Solids, 30(4), 559$570,2011$.

[7] Khalili, S.M.R., Mohammadi, Y., Free vibration analysis of sandwich plates with functionally graded face sheets and temperature-dependent material properties: A new approach. European Journal of Mechanics - A/Solids, 35, 61-74, 2012. 
[8] Sahoo, R., Singh, B.N., A new trigonometric zigzag theory for static analysis of laminated composite and sandwich plates. Aerospace Science and Technology, 35, 15-28, 2014.

[9] Thai, H.T., Nguyen, T.K., Vo, T.P., Lee, J., Analysis of functionally graded sandwich plates using a new first-order shear deformation theory. European Journal of Mechanics - A/Solids, 45, 211-225, 2014.

[10] Plagianakos, T.S., Papadopoulos, E.G., Higher-order 2-D/3-D layerwise mechanics and finite elements for composite and sandwich composite plates with piezoelectric layers. Aerospace Science and Technology, 40, 150-163, 2015.

[11] Natarajan, S., Haboussi, M., Manickam, G., Application of higher-order structural theory to bending and free vibration analysis of sandwich plates with CNT reinforced composite facesheets. Composite Structures, 113, 197-207, 2014.

[12] Kheirikhah, M.M., Khalili, S.M.R., Malekzadeh Fard, K., Biaxial buckling analysis of soft-core composite sandwich plates using improved high-order theory. European Journal of Mechanics A/Solids, 31, 54-66, 2012.

[13] Ghayesh, M.H., Amabili, M., Pai"doussis, M.P., Nonlinear dynamics of axially moving plates. Journal of Sound and Vibration, 332, 391-406, 2013.

[14] Dong Yang, X., Qun Chen, L., Zu, J.W., Vibrations and stability of an axially moving rectangular composite plate. Journal of Applied Mechanics, 78, 011018-011029, 2010.

[15] Hatami, S., Ronagh, H.R., Azhari, M., Exact free vibration analysis of axially moving viscoelastic plates. Computers \& Structures, 86, 1738-1746, 2008.

[16] Marynowsky, K., Free vibration analysis of the axially moving Levy-type viscoelastic plate. European Journal of Mechanics - A/Solids, 29, 879-886, 2010.

[17] Marynowski, K., Grabski, J., Dynamic analysis of an axially moving plate subjected to thermal loading. Mechanics Research Communications, 51, 67-71, 2013.

[18] Marynowski, K., Dynamic analysis of an axially moving sandwich beam with viscoelastic core. Composite Structures, 94, 2931-2936, 2012.

[19] Bozhevolnaya, E., Sun, J.Q., Free vibration analysis of curved sandwich beams. Journal of Sandwich Structures and Materials, 6, 47-73, 2004.

[20] Reddy, J.N., Mechanics of Laminated Composite Plates and Shells: Theory and Analysis, CRC Press, 2004.

[21] Ghorbanpour Arani, A., Haghparast, E., Heidari Rarani, M.H., Khoddami Maraghi, Z., Strain gradient shell model for nonlinear vibration analysis of visco-elastically coupled Boron Nitride nanotube reinforced composite micro-tubes conveying viscous fluid. Computational Materials Science, 96, 448-458, 2015.

[22] Wang, X.Z., Shen, S.H., Nonlinear vibration and bending of sandwich plates with nanotubereinforced composite face sheets. Composite Part B: Engineering, 43, 411-421, 2012. 